\title{
Theoretical study of electrode materials for sodium ion batteries based on Graphether/Graphene heterostructure
}

\author{
Lei $\mathrm{Li}^{1}$ and Chun-Sheng $\mathrm{Liu}^{1, *}$ \\ ${ }^{1}$ College of Electronic and Optical Engineering, Nanjing University of Posts and Telecommunications, Nanjing 210023, China
}

\begin{abstract}
The graphether/graphene vdW heterostructure has been systematically studied as an electrode material for sodium batteries based on density functional theory. We predict that the graphether/graphene heterostructure exhibits low diffusion barrier and large capacity. All these calculation results suggest that the graphether/graphene heterostructure can be used as a future commercial anode material for sodium ion batteries.
\end{abstract}

\section{Introduction}

The demand for lithium ion batteries has increased since the commercialization of actual portable electronic devices such as mobile phones and notebook computers ${ }^{[1,2]}$. However, lithium storage in nature is very limited and lithium batteries also have some safety concerns, making it urgent to find lower-cost alternatives of lithium batteries. Sodium batteries have attracted the interest of researchers due to the abundance of sodium reserves in natural resources and the fact that sodium and lithium are very similar in physical and chemical properties, which means that the sodium batteries can be an alternative to lithium batteries and the mature technology of lithium batteries can be directly applied in sodium batteries ${ }^{[3]}$. However, the lack of novel highperformance anode materials has hindered the development and application of sodium batteries.

In the past decade, the traditional anode material graphite has been widely commercialized as the most suitable anode material for lithium-ion batteries due to its low cost, cyclic stability and high energy stability ${ }^{[4,5]}$. However, the low specific capacity $\left(372 \mathrm{mAhg}^{-1}\right)$ hinders its application in future battery electrodes. Nevertheless, traditional anode materials, graphite and silicon, are not suitable for sodium batteries ${ }^{[6]}$. It has been reported that graphene, as a typical well known two-dimensional material, exhibits high capacity and low migration barrier in lithium-ion batteries and has been used as an anode material ${ }^{[7-10]}$, however, lithium exhibits weaker forces in combination with graphene and certain two-dimensional materials, thus impeding their application in lithium-ion batteries ${ }^{[1-12]}$. To date, the demand for a new generation of batteries based on twodimensional materials with higher power capacity, good cycle stability, and longer life continues to grow. However, the adsorption of $\mathrm{Na}$ ions on $2 \mathrm{D}$ materials leads to large volume changes, which can lead to severe capacity degradation. It has been shown that the design of 2D van der Waals heterostructures using different isolated 2D materials stacked vertically can effectively suppress the deformation of the structure caused by the charge and discharge process ${ }^{[13-15]}$. In consideration of the structural stability of the electrode material, graphene with high stability, flexibility and excellent conductive properties can be integrated with other $2 \mathrm{D}$ materials to solve the structural deformation and storage performance challenges ${ }^{[16-18]}$. It is reported recently that the novel 2D material graphether exhibits excellent electron mobility, high stability and adjustable direct band gap, and its puckered surface provides more space to ensure a stable sodium storage capacity ${ }^{[19]}$.

Up to now, the systematical research of graphether/graphene vdW heterostructure as an anode material for sodium ion batteries is not clear so far. In our study, the adsorption and diffusion behaviors of $\mathrm{Na}$ ions on graphether/graphene heterostructures based on density functional theory (DFT) have been investigated systematically. All these features suggest that graphether/graphene vdW heterostructures can be one of the candidate anode materials for future sodium batteries.

\section{Computational method}

All physical properties were carried out with density functional theory method as implemented in the Cambridge Sequential Total Energy Package ${ }^{[20]}$. The exchange and correlation energies are adopted with the generalized gradient approximation (GGA) parameterized by Perdew, Becke and Ernzerhof $(\mathrm{PBE})^{[21]}$. The weak van der Waals interacting systems were taken into account by using Tkatchenko and Scheffler approaches of the DFT-D method ${ }^{[22]}$. The convergences tolerances for the maximum force, maximum displacement and energy were set $5.0 \times 10^{-4} \mathrm{Ha} / \AA$, $5.0 \times 10^{-4} \AA$ and $1.0 \times 10^{-7} \mathrm{Ha}$, respectively. To eliminate the interactions between periodic images, the sufficient vacuum region is taken to be $30 \AA$. The Brillouin zone is

$\overline{{ }^{*} \text { Corresponding author: csliu@njupt.edu.cn }}$ 
sampled using a $2 \times 7 \times 1$ Monkhorst-Pack grid with 0.02 $\AA^{-1}$ separation. The Na ion migration was calculated by diffusion barrier and transition state search, we use the quadratic synchronous transit (LST/QST) method and the TS is confirmed by the nudged elastic band (NEB) method $^{[23]}$.

\section{Results and discussion}

\subsection{Structure of the Graphether/Graphene bilayer heterostructure}

Initially, the graphether and graphene sheets were performed by full geometry optimization. According to the calculation result, in order to keep the lattice mismatch within $5 \%$, we stack $6 \times 1$ graphether supercell and $5 \times 1$ graphene monolayer supercell to form graphether/graphene heterostructure. The in-plane lattice constants of the combined system unit cell are $21.36 \AA$ and $7.40 \AA$ along the armchair and zigzag direction.

The small lattice mismatch are approximately $1.6 \%$ and $4.5 \%$ along the armchair and zigzag directions, which means that slightly compress graphether in different directions to fit to graphene supercell. Layers between adjacent layers tend to slide in heterostructure, therefore, we keep the lattice of graphene fixed and slide the graphether system along different directions. In order to explore the stability of bilayer heterostructure, eight possible stacking patterns are considered. (a)

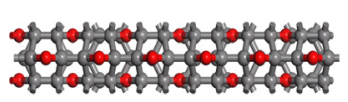

(c)

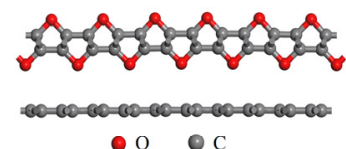

- $\mathrm{O}$

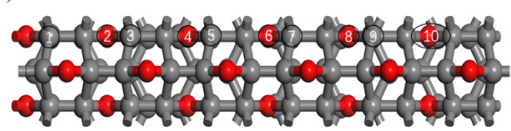

Figure 1. Top and side views of the graphether/graphene heterostructure shown as (a) and (b), respectively. (c) Top views of the possible absorption sites on the graphether monolayer. Site 1, 3, 5, 7, 9 are on the top of $\mathrm{C}$ atoms and site $2,4,6,8,10$ are on the top of the $\mathrm{O}$ atoms.

Comparing the calculation results of eight stacking of graphether/graphene bilayer heterostructure, the stable structural configuration of the graphether/graphene heterostructure was determined as shown in Figure 1. After full geometric optimization, the interlayer spacing between graphether and graphene monolayer is about $2.995 \AA$, which is consistent with other van der Waals heterostructure. To evaluate the stacking stability of the graphether/graphene heterostructure system, the formation energy $(\mathrm{Eb})$ is calculated according to the equation:

$E_{b}=\left(E_{\text {graphether/graphene }}-E_{\text {graphether }}-E_{\text {graphene }}\right) / N$

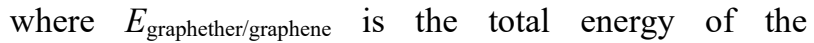
heterostructure, $E_{\text {graphether }}$ and $E_{\text {graphene }}$ denote the energies of isolated layers of graphether and graphene, and $N$ denotes the total number of atoms in the supercell. The calculated formation energy is about $-23 \mathrm{meV}$ per atom, which is comparable to the values with other 2D graphene based vdW crystals for $\mathrm{MoS}_{2} / \mathrm{G}^{[24]}, \mathrm{BP} / \mathrm{G}^{[25]}$ and $\mathrm{SiC} / \mathrm{G}^{[26]}$, etc. The negative binding energy and rational lattice match imply the structure stability of the graphether/graphene heterostructure.

\section{2. $\mathrm{Na}$ atom adsorption}

The electrochemical properties of electrode materials depend on the $\mathrm{Na}$ capacity and the firm ability of $\mathrm{Na}$ ions to adsorb to the electrode surface.

To further explore the adsorption properties of $\mathrm{Na}$, three types of adsorption sites were considered and the heterostructure was fully relaxed to allow the $\mathrm{Na}$ atoms to find stable adsorption sites. First is placed on the outside surface of graphether $(\mathrm{Na} / \mathrm{G} / \mathrm{G})$, the second is beneath the $\mathrm{O}$ atom in the sandwich between graphether and graphene $(\mathrm{G} / \mathrm{Na} / \mathrm{G})$, and the last is the hollow site located directly above the center of the graphene C6 ring $(\mathrm{G} / \mathrm{G} / \mathrm{Na})$. All the stable adsorption sites of $\mathrm{Na}$ on the surface of graphether are shown as in Figure 1 (c) and the corresponding adsorption energies are listed in Table 1. In Table 1, we list the calculated adsorption energies of the $\mathrm{Na}$ ions at different adsorption sites, and the adsorption energy of sodium on the graphether/graphene heterostructure can be calculated as follows:

$$
E_{\text {ad }}=E_{\text {graphether/graphene }+\mathrm{Na}}-E_{\text {graphether/graphene }}-E_{\mathrm{Na}}(2)
$$

According to the definition of the above equation, where $E_{\text {graphether/graphene+Na }}$ is the energy of the heterostructure after $\mathrm{Na}$ adsorption on the grapethere/graphene heterostructure, and $E_{\text {graphether/graphene }}$ is the total energy of the pristine graphether/graphene heterostructure. $E_{\mathrm{Na}}$ is the total energy of an isolated $\mathrm{Na}$ atom.

From Table 1, it can be seen that the most stable point of $\mathrm{Na}$ adsorption is site 3 , followed by metastable adsorption site 1 and site 5 . The adsorption energy above the $\mathrm{O}$ atom of the graphether is higher than that above the adjacent $\mathrm{C}$ atom, for example, site 2 and site 4 has an adsorption energy of $-0.68 \mathrm{eV}$, which is $0.11 \mathrm{eV}$ higher than that of site 3 , indicating that $\mathrm{Na}$ ions prefer to occupy above the $\mathrm{C}$ atom on the surface of the graphether.

When $\mathrm{Na}$ is adsorbed in the interlayer of graphether/graphene, the energy of adsorption is lowest at site 1 below the $\mathrm{O}$ atom, which is the most stable site between the monolayer graphether and graphene. It is clear from Table 1 that the adsorption energies of all interlayer adsorption sites are lower than those of the graphether and graphene surfaces. The adsorption of $\mathrm{Na}$ ions between the graphether/graphene heterostructure interlayer is superior to their adsorption with graphether and graphene surfaces. The stability of the $\mathrm{Na}$ ions between the interlayer is affected by the joint action of both graphether and graphene. 
Table 1. Binding energy $\left(E_{\mathrm{b}}\right)$ of absorbed one $\mathrm{Na}$ atom on the graphether/graphene heterostructure.

\begin{tabular}{ccc}
\hline & Na site & $E_{\mathrm{b}}$ \\
\hline $\mathrm{Na} / \mathrm{G} / \mathrm{G}$ & Site1 & $-0.69 \mathrm{eV}$ \\
& Site2 & $-0.68 \mathrm{eV}$ \\
& Site3 & $-0.79 \mathrm{eV}$ \\
& Site4 & $-0.68 \mathrm{eV}$ \\
& Site5 & $-0.69 \mathrm{eV}$ \\
Site6 & $-0.68 \mathrm{eV}$ \\
& Site7 & $-0.68 \mathrm{eV}$ \\
& Site8 & $-0.66 \mathrm{eV}$ \\
& Site9 & $-0.68 \mathrm{eV}$ \\
& Site10 & $-0.67 \mathrm{eV}$ \\
& & \\
& Site1 & $-1.20 \mathrm{eV}$ \\
$\mathrm{G} / \mathrm{Na} / \mathrm{G}$ & Site2 & $-1.12 \mathrm{eV}$ \\
& Site3 & $-1.04 \mathrm{eV}$ \\
& Site4 & $-1.05 \mathrm{eV}$ \\
& Site5 & $-1.04 \mathrm{eV}$ \\
& Site6 & $-0.25 \mathrm{eV}$ \\
& Site1 & \\
& Site2 & $-0.25 \mathrm{eV}$ \\
& Site3 & $-0.25 \mathrm{eV}$ \\
& Site4 & $-0.35 \mathrm{eV}$ \\
& Site5 & $-0.25 \mathrm{eV}$ \\
& & $-0.25 \mathrm{eV}$ \\
\hline
\end{tabular}

Obviously, these findings indicate that during the process of charging and discharging, sodium atoms preferentially move between graphether and graphene, rather than the outside surface of graphether and graphene. The implication is that sodium atoms first occupy the interlayer of graphether/graphene heterojunction and then take the outside surfaces of graphether and graphene.

(a)

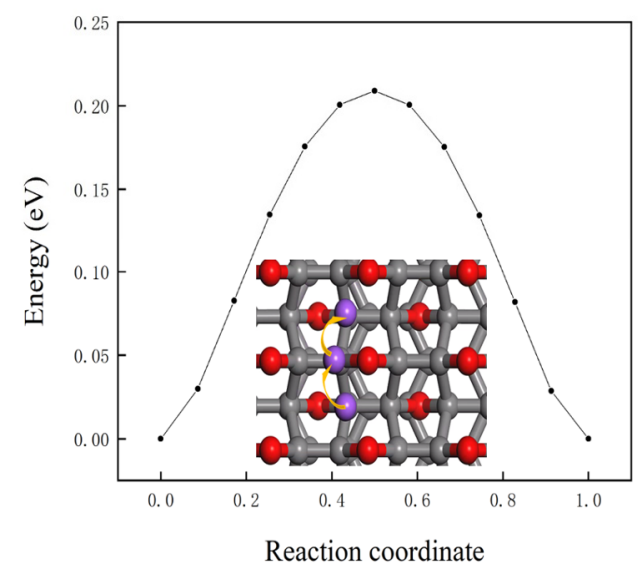

\subsection{Diffusion Properties}

The charge/discharge rate is also an important factor in determining the electrode material available for sodium batteries, which depends on the diffusion rate of $\mathrm{Na}$ atoms on the electrode material.

The $\mathrm{Na}$ diffusion on the graphether/graphene heterostructure occurs in three cases: (1) the diffusion of $\mathrm{Na}$ atoms on the outside surface of the graphether in the graphether/graphene heterostructure, (2) the diffusion of $\mathrm{Na}$ atoms in the interlayer of the graphether/graphene heterostructure, and (3) the diffusion of $\mathrm{Na}$ atoms on the surface of the graphene in the graphether/graphene heterostructure. As is shown in Figure 2 (a), we first considered various possible diffusion paths for $\mathrm{Na}$ atoms on the surface of graphether in graphether/graphene heterostructure, with site 3 being the most stable adsorption site when $\mathrm{Na}$ is adsorbed on the graphether surface, and the possible diffusion paths for moving from site 3 to adjacent site 3 , namely $3->\mathrm{S}->3$. Although the $\mathrm{Na}$ atoms move in the adjacent position 3 , the entire diffusion process is within the groove of the graphether, and the corresponding graphether surface diffusion barrier is $0.21 \mathrm{eV}$. It has been calculated that the diffusion energy barrier of $\mathrm{Na}$ atoms on pristine graphene is determined to be $0.11 \mathrm{eV}^{[27]}$, and our calculations indicating that the diffusion energy barrier of $\mathrm{Na}$ on the graphene surface is $0.10 \mathrm{eV}$, which is decreased about $0.01 \mathrm{eV}$ lower than that of pristine graphene. The calculation that $\mathrm{Na}$ ions prefer to diffuse from hollow sites to adjacent hollow sites on pristine graphene has been reported, which is consistent with our adsorption results on the outside surface of graphene in the graphether/graphene heterostructure.

(b)

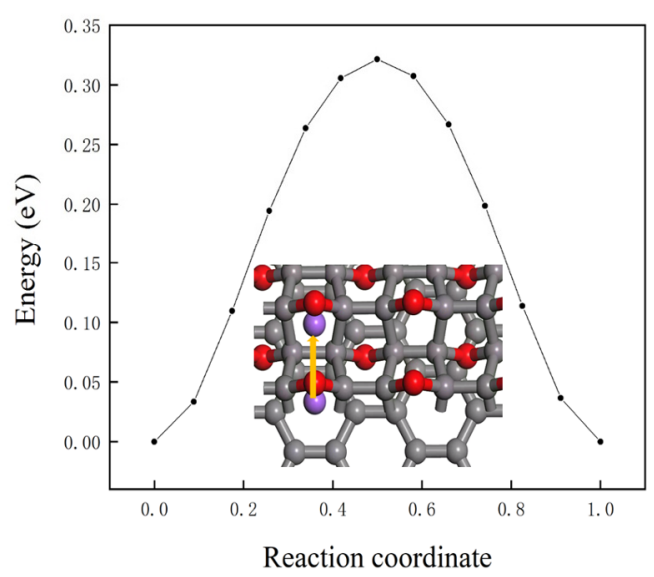

Figure 2. The energy barrier for Na diffusing on the surface of the graphether is shown as (a). The energy barrier for Na diffusing in the interlayer of the graphether/graphene heterostructure is shown in (b).

This means that the fast charge/discharge capability properties of graphene are well preserved in the graphether/graphene heterostructure. Next, the diffusion behavior of $\mathrm{Na}$ between the graphether/graphene heterostructure interlayer was also investigated. Our calculations show that the value of the $\mathrm{Na}$ diffusion barrier $(1->\mathrm{S}->1)$ between the interlayer $(0.32 \mathrm{eV})$ shown in Figure 2 (b) is significantly higher than that of the barrier on the graphether $(0.21 \mathrm{eV})$ and graphene $(0.10 \mathrm{eV})$ surfaces, which may be attributed to the action of graphether and graphene collectively. Thus, the predicted migration rate of $\mathrm{Na}$ atoms in the graphether/graphene heterostructure can be satisfied for the sodium batteries.

In addition to the low diffusion barrier, the storage capacity of the sodium battery electrode material is also 
an important factor in measuring its performance. The maximum theoretical specific capacity $\mathrm{C}$ is calculated using the following formula:

$$
C=\frac{x_{\max } \times Z \times F \times 10^{3}}{M_{\left(\text {graphether/graphene) } N a_{x}\right.}}
$$

where $F$ is the Faraday constant $(26.810 \mathrm{Ah} / \mathrm{mol}), Z$ is the valence number $(Z=1$ for $\mathrm{Na}), x_{\max }$ is the maximum number of $\mathrm{Na}$ atoms inserted, $M_{\text {(graphether/graphene)Nax }}$ is the atomic mass of the graphether/graphene heterostructure after absording the $\mathrm{Na}$ atoms. The graphether/graphene heterostructure accommodates up to $21 \mathrm{Na}$ atoms (the ratio of $\mathrm{C}: \mathrm{O}: \mathrm{Na}$ is $44: 12: 21$ ) without obvious structure distortion and the maximum theoretical capacity of the graphether/graphene heterostructure for $\mathrm{Na}$ storage is predicted to reaches $468 \mathrm{mAhg}^{-1}$.

\section{Conclusions}

In summary, we have systematically explored the feasibility of graphether/graphene heterostructure as an anode material for sodium batteries based on the firstprinciples calculations. Our results show that up to $21 \mathrm{Na}$ atoms can be adsorbed in the graphether/graphene heterostructure, which indicates a high theoretical capacity. In fact, the capacity of the graphether/graphene heterostructure $\left(468 \mathrm{mAhg}^{-1}\right)$ is also greatly improved compared to the pristine graphene, and the lower diffusion barrier $(0.10 \mathrm{eV})$ on the graphene surface implies that the graphether/graphene heterostructure is more suitable for storing $\mathrm{Na}$ atoms than the pristine graphene.

Overall, the graphether/graphene heterostructure has a high capacity and excellent $\mathrm{Na}$ mobility, so the graphether/graphene heterostructure is expected to be applied to future electrode materials for sodium batteries, and these findings are of great significance for the understanding and application of graphene-based heterostructures and the rational design of anode materials for sodium batteries.

\section{Acknowledgements}

This work is supported by the National Natural Science Foundation of China (Grant No. 61974068)

\section{References}

1. Y. Wang, G. Cao, Master. Adv 20, 12 (2008)

2. G. Derrien, J. Hassoun, S. Panero, B. Scrosati, Adv. Mater 19, 17 (2007)

3. D. Kundu, E. Talaie, V. Duffort, et al., Angew Chem. Int. Ed. Engl 46, 21 (2015)

4. D. Aurbach, E. Zinigrad, Y. Cohen, H. Teller, Solid State Ionics 148, 3 (2002)

5. M. Yoshio, H. Y. Wang, K. Fukuda, Angew. Chem 115, 35 (2003)

6. M. M. Doeff, Y. Ma, S. J. Visco, et al., [J]. ChemInform 140, 12 (1994)
7. X. Lv, W. Wei, C. Mu, B. Huang and Y. Dai, J. Mater. Chem. A, 6, (2018)

8. W. Wei, Y. Dai and B. Huang, J. Mater. Chem. A, 5, (2017)

9. S. Lin, J. Gu, Y. Wang, Y. Wang, S. Zhang, X. Liu, H. Zeng and Z. Chen, J. Mater. Chem. A, 6, (2018)

10. Y. Jing, Z. Zhou, C. R. Cabrera and Z. Chen, J. Mater. Chem. A, 2, (2014)

11. E. Pollak, B. Geng, K.-J Jeon, I. T. Lucas, T. J. Richardson, F. Wang, R. Kostecki, Nano Lett, 10, (2010)

12. X. Fan, W. T. Zheng, J.-L Kuo, ACS Appl. Mater. Interfaces, 4, (2012)

13. J.-.H. Chen, L. Li, W.G. Cullen, E.D. Williams, M.S. Fuhrer, Nat. Phys, 7, (2011)

14. G. C. Guo, D. Wang, X. Wei, et al., J. Phys. Chem. Lett 6, 24 (2015)

15. S. Shahriari, N.S. Soofi, F. Farzi, et al., J. Comput. Theor. Nanosci 13, 5 (2016)

16. D. Wang, D. Choi, J. Li, Z. Yang, Z. Nie, R. Kou, D. Hu, C. Wang, L. V. Saraf, J. Zhang, ACS Nano 3, 4 (2009)

17. V. C. Tung, L.-M Chen, M. J. Allen, J. K. Wassei, K. Nelson, R. B. Kaner, Y. Yang, Nano Lett 9, 5 (2009)

18. H. Jiang, J. Ma, C. Li, Adv. Mster 24, 30 (2012)

19. G. L. Zhu, X. J. Ye and C. S. Liu, Nanoscale, 11, (2019)

20. M. D. Segall, P. J. D. Lindan, M. J. Probert, C. J. Pickard, P. J. Hasnip, S. J. Clark and M. C. Payne, J. Phys.: Condens. Matter, 14, (2002)

21. J. P. Perdew, K. Burke and M. Ernzerhof, Phys. Rev. Lett, 77, (1996)

22. A. Tkatchenko and M. Scheffler, Phys. Rev. Lett, 102, (2009)

23. N. Govind, M. Petersen, G. Fitzgerald, D. KingSmith and J. Andzelm, Comput. Mater. Sci 28, 8 (2003)

24. X. Shao, K. Wang, R. Pang, J. Phys. Chem. C 119, $46(2015)$

25. G. C. Guo, D. Wang, X. L. Wei, J. PHYS. CHEM. LETT 6, 24 (2015)

26. S. Li, M. Sun, J. P. Chou, Phys. Chem. Chem. Phys 20, 38 (2018)

27. K. Fan, T. Tang, S. Wu, et al, INT. J. MOD. PHYS. B 32, 1 (2018) 